\title{
Submitted: Diagnostic imaging of gastrointestinal neuroendocrine Accepted: neoplasms with a focus on ultrasound
} 26.06.2019

Published: 30.09.2019

Keywords

diagnostic imaging, ultrasound, neuroendocrine tumours, elastography

\author{
Joanna Walczyk ${ }^{1,2}$, Anna Sowa-Staszczak $k^{1,2}$ \\ ${ }^{1}$ Clinical Department of Endocrinology, University Hospital in Kraków, Kraków, Poland \\ 2 Jagiellonian University, Medical College, Faculty of Medicine, Kraków, Poland \\ Correspondence: Joanna Walczyk, Oddziat Kliniczny Kliniki Endokrynologii, \\ Szpital Uniwersytecki, ul. Kopernika 17, 31-501 Kraków, Poland. Tel. +48 664989 096, \\ e-mail: joasia.walczyk@gmail.com
}

DOI: $10.15557 / J o U .2019 .0034$

\begin{abstract}
The diagnosis of gastrointestinal neuroendocrine neoplasms represents a significant diagnostic challenge since these tumours have a various, often non-specific clinical presentation. Currently, more than half of gastroenteropancreatic neuroendocrine neoplasms are detected incidentally, usually during surgery, diagnostic imaging studies or endoscopic procedures performed for other indications. Sometimes the first symptom of the disease is the presence of metastatic lesions in the liver. A neuroendocrine tumour is diagnosed based on the clinical presentation, assessment of specific and non-specific biochemical markers, imaging studies and histopathological examination. Focal lesions, both primary and metastatic may be small and often have an atypical location. Diagnostic imaging of neuroendocrine tumours is of fundamental importance for determining the location of the primary lesion, staging of the disease, selection of treatment and monitoring of its effects. In addition, diagnostic imaging make it possible not only to detect tumours, but also to perform therapeutic procedures based on the result. Transabdominal ultrasound is one of the first diagnostic imaging method for neuroendocrine neoplasms. New ultrasound techniques such as ultrasound elastography, contrast-enhanced ultrasound, endoscopic ultrasound, intraductal and intraoperative ultrasound improve the efficacy of ultrasound examination. Endoscopic ultrasound is a fundamental diagnostic tool for the detection of neuroendocrine tumours of the pancreas and the distal part of the colon. Due to the large variety of neuroendocrine tumours and differences in tumour biology, clinical stage and expression of somatostatin receptors, no single imaging method is sufficient; therefore, in order to determine the right diagnosis and select the best treatment, it is recommended that a combined morphological and functional assessment be
\end{abstract} used. 


\section{Introduction}

Diagnostic imaging of gastrointestinal neuroendocrine neoplasms (NEN) is of fundamental importance for determining the location of the primary lesion, disease staging, selection of treatment and monitoring its effects. Currently, more than half of gastrointestinal NEN are detected incidentally, usually during surgery, diagnostic imaging studies or endoscopic procedures performed for other indications $^{(1,2)}$. Sometimes metastatic lesions in the liver are the first sign of the disease when the primary lesion is not yet known ${ }^{(1)}$. Focal lesions, both primary and metastatic may be small and often have an atypical location, which makes NEN imaging and diagnosis difficult.

\section{Gastroenteropancreatic neuroendocrine neoplasms}

Gastroenteropancreatic neuroendocrine neoplasms (GEPNEN) account for approximately $70 \%$ of neuroendocrine neoplasms and approximately $1.5-2 \%$ of all gastrointestinal and pancreatic neoplasms ${ }^{(1,2)}$. GEP-NEN arise from diffuse endocrine system (DES) cells present in the pancreas and the gastrointestinal system ${ }^{(1,3)}$. Neuroendocrine tumours of the pancreas account for $7 \%$ of all GEP-NEN and approximately $10 \%$ of all pancreatic neoplasms ${ }^{(4)}$. In recent years an increase in the prevalence of gastric and rectal NEN versus intestinal NEN has been observed ${ }^{(1,5-10)}$. The NEN detection rate has significantly increased over the last few decades $^{(1,3)}$. Currently, it is estimated that NEN incidence is $3-5.2 / 100,000$ new cases per year. The general prevalence rate for these neoplasms is $35 / 100,000$ cases on average, i.e. 5.86/100,000 individuals per year ${ }^{(1,3)}$. The prevalence of NEN in men is higher than that in women $(52 \%$; $5.35 / 100,000 /$ year vs $48 \%$; $4.76 / 100,000 /$ year $)^{(1,3)}$. A higher risk of the disease has been noted in African Americans, Latinos and Asians and the mean age upon diagnosis is 63 years $^{(5,6)}$. The majority of NEN are sporadic in nature and develop approximately 15 years later than the rarer NEN associated with genetic syndromes $(25 \%)^{(4,7)}$. Pancreatic neuroendocrine neoplasms (mainly hormonally inactive ones) and gastrinoma occur in 25-75\% patients with MEN1 syndrome ${ }^{(7)}$. In patients with a mutation in the VHL gene, neuroendocrine tumours are usually benign, while other neoplasms occurring in this syndrome (e.g. clear cell renal cell carcinoma) are malignant ${ }^{(7,8)}$. Pancreatic neuroendocrine neoplasms in NF1 syndrome and tuberous sclerosis complex are rare (1\%); the diagnosis is made in late-stage disease $^{(9)}$.

In the majority of GEP-NEN cases no risk factor for the development of a tumour was identified. It was observed that hypergastrinaemia, diabetes and ulcerative colitis may be associated with the development of NEN, but no causeand-effect relationship was confirmed ${ }^{(4)}$.

A special feature of NEN is the production of hormones or biogenic amines which can produce numerous clinical symptoms. In the case of disease spread symptoms of carcinoid syndrome may occur such as diarrhoea, tachycardia, hot flushes and reddening of the skin ${ }^{(3)}$. The majority of NEN are hormonally inactive with uncharacteristic symptoms associated mainly with the local development of a tumour (abdominal pain, compression, ileus) or spread to the liver ${ }^{(4)}$.

Currently, more than half of GEP-NEN are detected incidentally, usually during surgery of the small intestine and appendix and in imaging studies or endoscopic procedures performed for other indications ${ }^{(1,4)}$. Sometimes the first symptom of the disease is metastatic lesions in the liver ${ }^{(1,4)}$. The proportion of small intestinal NEN in which the primary lesion was not found is approximately $70 \%$. The 5-year survival rate for these patients is estimated to be $50 \%{ }^{(10)}$.

A neuroendocrine tumour is diagnosed based on clinical presentation, assessment of specific and non-specific NEN markers, imaging studies and histopathological examination $^{(4)}$. As for laboratory testing, assay of chromogranin A (CgA), which is a characteristic, but non-specific marker, may be helpful. The monitoring of changes in the levels of this marker may be very helpful for excluding recurrence or spread of the neoplastic process, particularly for NEN arising from the small intestine ${ }^{(1)}$. Other markers assessed depending on the symptoms presented by the patient and the suspected type of NEN include: chromogranin B, pancreatic polypeptide, gastrin, 5-hydroxyindoleacetic acid (5-HIAA), glucose, insulin, C-peptide, glucagon and vasoactive intestinal peptide (VIP). If MEN1 is suspected, the assessment of pituitary function, evaluation of calcium and phosphate balance and genetic testing are necessary. A diagnostic novelty is NETest with the assessment of expression of selected gene transcripts characteristic for NEN. The evaluation of circulating microRNA, which are stable in body fluids and characteristic for a given neoplasm, is a promising method $\mathrm{d}^{(1,11)}$.

Many different classifications have been proposed in the history of neuroendocrine neoplasms. The most recent one, adopted by the World Health Organisation (WHO) in 2017, introduced a division according to the histological grade of the neoplasm (the $G$ feature) and the proliferation index (Ki-67) into four groups: NEN G1, G2 and G3 and NEC (neuroendocrine carcinoma ${ }^{(1)}$.

The primary method of GEP-NEN management is surgery, whose scope depends on the location of the tumour, the stage of the neoplasm and the patient's condition. In selected cases endoscopic treatment is an alternative method $^{(1)}$. The gold standard of pharmacotherapy in patients with NEN is somatostatin analogue treatment for hormonally active and inactive tumours, both during stable disease and progression ${ }^{(1)}$. Another NEN treatment method is chemotherapy, which is selected depending on the stage and grade of the disease. For NEN G3 a less aggressive capecitabine and temozolomide (CAPTEM) regimen can be used, while for NEC cisplatin and etoposide are used ${ }^{(1)}$. Molecularly targeted therapies (everolimus and sunitinib) are offered particularly for pancreatic $\mathrm{NEN}^{(1)}$. In patients with metastatic or inoperable NEN with high somatostatin 
receptor expression on somatostatin receptor scintigraphy (SRS), treatment with radiolabelled somatostatin analogues (DOTA-Tyr3-octreotide and DOTA-Tyr3-octreotate labelled with radioisotopes ${ }^{177} \mathrm{Lu},{ }^{90} \mathrm{Y}$ ) is recommended, which is called peptide receptor radionuclide therapy $(\mathrm{PRRT})^{(1)}$.

\section{Diagnostic imaging of NEN}

Diagnostic imaging of neuroendocrine tumours plays a fundamental role in diagnosis, staging, selection of treatment method, assessment for radioisotope therapy and monitoring response to treatment ${ }^{(1,2)}$. Diagnostic imaging methods make it possible not only to detect tumours, but also to perform therapeutic procedures based on the result. Diagnostic imaging of NEN is difficult since the tumours have a various, often non-specific clinical presentation or are diagnosed incidentally in asymptomatic patients. Focal lesions, both primary and metastatic may be small and often have an atypical location. It is important to use various diagnostic methods, both morphological and functional. Morphological imaging (transabdominal ultrasound, contrast-enhanced ultrasound, [CEUS]; elastography; endoscopic ultrasound [EUS]; computed tomography [CT]; magnetic resonance imaging [MRI]; endoscopy, including video capsule endoscopy [VCE]) is usually used to detect a tumour and assess the stage of the disease. Functional imaging techniques (scintigraphy with ${ }^{99 \mathrm{~m}} \mathrm{Tc}$-labelled somatostatin analogues [ ${ }^{99 \mathrm{~m} T c-S P E C T}$; positron emission tomography with ${ }^{68} \mathrm{Ga}$-labelled peptides [68Ga-PET/CT] and with other tracers: ${ }^{18} \mathrm{~F}-\mathrm{FDG},{ }^{18} \mathrm{~F}$-DOPA and ${ }^{11} \mathrm{C}$-HTP) are useful for tumour detection, prognostic assessment and treatment planning ${ }^{(4)}$ (Tab. 1).

\section{Transabdominal ultrasound in NEN}

Transabdominal ultrasound is usually a first-line diagnostic imaging procedure for GEP-NEN. The use of various ultrasound techniques: harmonic and Doppler imaging, computer processing and compound imaging (CI) makes ultrasound a commonly used method for the diagnosis and monitoring of NEN. It is also possible to perform ultrasound-guided biopsy of tumours located in the liver, pancreas and retroperitoneal space.

The sensitivity of this method depends on the location of abnormalities, anatomical conditions, experience of the physician performing the procedure and technical aspects of the ultrasound device ${ }^{(1)}$. The sonographic image of NEN, both the primary lesion and liver metastases is non-characteristic; it depends on the type of NEN, proliferation index, tumour size and location and on the presence of regressive changes $^{(1)}$.

A pancreatic neuroendocrine neoplasm is often welldefined, hypoechoic (less commonly hyper- or normoechoic), sometimes with a hyperechoic halo, areas of necrosis and calcifications ${ }^{(1,4)}$.
Transabdominal ultrasound has limited utility for detecting a primary lesion in the small intestine. It is rarely possible to detect a lesion in the intestinal wall using high-frequency probes $^{(12)}$. A case of small intestinal tumour diagnosis with abdominal ultrasound despite negative results of other imaging studies has been reported in the literature ${ }^{(10)}$. Secondary lesions in the mesentery (enlarged, hypoechoic lymph nodes, mesenteric fibrosis) may be an indirect indication of a tumour in the intestinal wall(12).

Hepatic metastases of a primary NEN tumour located in the lungs, stomach or colon are usually hypo- or isoechoic compared to the hepatic parenchyma. In tumours arising from the small intestine and pancreas the echogenicity of secondary lesions is various and non-characteristic (hypo-, iso-, hyperechoic or sometimes mixed lesions). In insulinoma tumours, liver metastases usually have decreased echogenicity ${ }^{(13-15)}$. Areas of necrosis are usually found in large tumours $(>3 \mathrm{~cm})$ with a $\mathrm{Ki}-67$ proliferation index of $>2 \%$ and in individuals who have undergone treatment (interferon, embolisation, chemotherapy, radiofrequency ablation). No necrotic changes were observed in patients treated with cold or hot somatostatin analogues ${ }^{(13-15)}$.

On Doppler ultrasound, primary NEN foci and liver and lymph node metastases have a rich network of blood vessels. In tumours with $\mathrm{Ki}-67>2 \%$ the vascular network may be irregular ${ }^{(13-15)}$.

The sensitivity of transabdominal ultrasound in the diagnosis of metastatic lesions in the liver is $82-88 \%$ and its specificity is $92-95 \%$. The sensitivity for pancreatic tumours is lower: $39 \%$.

\section{New ultrasound techniques in the diagnosis of NEN}

In recent years new ultrasound imaging techniques have been developed that improve the efficacy of NEN diagnosis $^{(15)}$ : ultrasound elastography, CEUS, EUS, intraductal ultrasound (IDUS) and intraoperative ultrasound (IOUS).

\section{Endoscopic ultrasound (EUS)}

Endoscopic ultrasound is an invasive imaging method which involves the insertion of a flexible endoscope with a high-frequency ultrasound probe (7.5-12 MHz), which is positioned near the pancreas, stomach, duodenum or rectum. The minimisation of the distance between the probe and the abnormality to be evaluated makes it possible to thoroughly assess anatomical relationships, depth of tumour invasion through the gastrointestinal wall and the presence of metastases in local lymph nodes ${ }^{(1)}$.

Endoscopic ultrasound is a fundamental diagnostic tool for the detection of NEN in the pancreas and the distal part of the colon. Combined with simultaneous fine-needle biopsy, it is the most sensitive method of pancreatic NEN diagnosis. It is a method of choice for the detection of small lesions 
Tab. 1. Comparison of selected diagnostic imaging methods for GEP-NEN $N^{(1,12,18,19)}$

\begin{tabular}{|c|c|c|}
\hline Method & Advantages & Disadvantages \\
\hline Ultrasound & $\begin{array}{l}\text { - No exposure to ionising radiation } \\
\text { - Repeatability } \\
\text { - Transabdominal ultrasound - wide availability } \\
\text { - EUS - primary method for pancreatic tumour assessment } \\
\text { (EUS + CEUS, elastography) } \\
\text { - EUS-FNA - possibility of cytological verification } \\
\text { - EUS-RFA - possibility to treat focal lesions of the pancreas } \\
\text { - CEUS, elastography - improved sensitivity for focal lesion } \\
\text { assessment, evaluation of unclear lesions on CT/MRI } \\
\text { - IOUS - possibility of intraoperative lesion assessment } \\
\text { - IDUS - thorough assessment of intraductal lesions }\end{array}$ & $\begin{array}{l}\text { - Assessment dependent on the skills and experience of the } \\
\text { examiner and the class of the device } \\
\text { - Poorer sensitivity of the classic method } \\
\text { - EUS, IOUS, IDUS, CEUS - access only at specialised centres, } \\
\text { invasive procedures }\end{array}$ \\
\hline $\begin{array}{l}\text { Computed } \\
\text { tomography }\end{array}$ & $\begin{array}{l}\text { - High spatial resolution (min. } 2-4 \mathrm{~mm} \text { ) } \\
\text { - Thorough anatomical assessment of abdominal organs } \\
\text { - Multiplanar imaging, 3D reconstruction } \\
\text { - Disease staging } \\
\text { - Assessment of intestinal focal lesions (enteroclysis, } \\
\text { enterography, CT colonoscopy) } \\
\text { - Aid in surgery planning } \\
\text { - Availability, quick results, repeatability }\end{array}$ & $\begin{array}{l}\text { - Exposure to ionising radiation } \\
\text { - Exposure to iodine contrast agent and the associated } \\
\text { complications (renal failure, allergic reactions, } \\
\text { hyperthyroidism) } \\
\text { - Vasculature assessment dependent on the phase and dose } \\
\text { of contrast } \\
\text { - Difficult reassessment of both small and too large lesions in } \\
\text { terms of volume } \\
\text { - Difficult assessment of response to treatment if necrosis, } \\
\text { haemorrhage or fibrosis are present with no reduction in } \\
\text { lesion size } \\
\text { - Difficult assessment in slowly growing lesions }\end{array}$ \\
\hline $\begin{array}{l}\text { Magnetic } \\
\text { resonance } \\
\text { imaging }\end{array}$ & $\begin{array}{l}\text { - High spatial resolution (min. } 2-4 \mathrm{~mm} \text { ) } \\
\text { - Best differentiation between soft tissues } \\
\text { - Multiplanar imaging, 3D reconstruction } \\
\text { - Disease staging } \\
\text { - The best method for the assessment of hepatic and } \\
\text { pancreatic focal lesions } \\
\text { - Assessment of bile duct and pancreatic duct - MRI } \\
\text { cholangiopancreatography } \\
\text { - No exposure to ionising radiation } \\
\text { - Gadolinium contrast - fewer allergic reactions, no kidney } \\
\text { damage } \\
\text { - Repeatability }\end{array}$ & $\begin{array}{l}\text { - High costs } \\
\text { - Limited availability } \\
\text { - Long duration of procedure } \\
\text { - Patient cooperation required } \\
\text { - Contraindication: metal parts in the body } \\
\text { - Difficult reassessment of both small and too large lesions in } \\
\text { terms of volume } \\
\text { - Difficult assessment of response to treatment if necrosis, } \\
\text { haemorrhage or fibrosis are present with no reduction in } \\
\text { lesion size } \\
\text { - Difficult assessment in slowly growing lesions }\end{array}$ \\
\hline${ }^{99} \mathrm{mTC}-\mathrm{SPECT}$ & $\begin{array}{l}\text { - Functional examination } \\
\text { - Full-body scan } \\
\text { - CT imaging possible } \\
\text { - Assessment of primary lesion location, stage of the disease } \\
\text { - Evaluation for appropriate forms of treatment, assessment of } \\
\text { treatment response, evaluation for PRRT } \\
\text { - Monitoring, reassessment } \\
\text {-1-day procedure, SPECT } 4 \text { hours after tracer administration }\end{array}$ & $\begin{array}{l}\text { - Exposure to ionising radiation } \\
\text { - Low resolution, poor assessment of lesions }<1 \mathrm{~cm} \\
\text { - High background hinders midgut NEN assessment on } \\
\text { gastrointestinal examination } \\
\text { - Low sensitivity in insulinoma detection } \\
\text { - Possible interference with cold somatostatin analogues }\end{array}$ \\
\hline${ }^{68} \mathrm{Ga}$-DOTA-PET & $\begin{array}{l}\text { - Functional examination } \\
\text { - Full-body scan } \\
\text { - Multiplanar imaging, high resolution 4-6 mm, imaging } \\
\text { together with CT } \\
\text { - Possibility to calculate the level of uptake - standardised } \\
\text { uptake value (SUV) } \\
\text { - Good anatomical assessment } \\
\text { - Assessment of primary lesion location, stage of disease, } \\
\text { evaluation for appropriate forms of treatment, assessment of } \\
\text { treatment response, monitoring, reassessment } \\
\text { - Assessment for PRRT } \\
\text { - 1-day procedure, images obtained quickly, after } 2 \text { hours }\end{array}$ & $\begin{array}{l}\text { - Uptake in normal tissues (pituitary gland, spleen, kidneys, } \\
\text { adrenal glands) or in inflammatory foci may be mistaken for } \\
\text { a tumour } \\
\text { - Possible interference with cold somatostatin analogues } \\
\text { - Lack of complete validation }\end{array}$ \\
\hline
\end{tabular}




\begin{tabular}{|c|c|c|}
\hline${ }^{18}$ FDG-PET & $\begin{array}{l}\text { - Functional examination } \\
\text { - Full-body scan } \\
\text { - Multiplanar imaging, high resolution } 4-6 \mathrm{~mm} \text {, imaging } \\
\text { together with CT } \\
\text { - Good anatomical assessment } \\
\text { - Disease staging, assessment of treatment response, } \\
\text { prognostic factor, monitoring } \\
\text { - For the assessment of poorly differentiated NEN } \\
\text { - Prognostic value in highly and medium differentiated NEN }\end{array}$ & $\begin{array}{l}\text { - Exposure to radiation } \\
\text { - Poor uptake in NEN G1 and G2 } \\
\text { - Procedure available in specialised centres }\end{array}$ \\
\hline${ }^{131}$ I-MIBG-SPECT & $\begin{array}{l}\text { - Functional examination } \\
\text { - Full-body scan } \\
\text { - High specificity for phaeochromocytoma, paraganglioma, } \\
\text { neuroblastomas } \\
\text { - Assessment for treatment with }{ }^{131} I-\text { MIBG }\end{array}$ & $\begin{array}{l}\text { - High background } \\
\text { - Poor anatomical assessment } \\
\text { - Interference from many pharmaceuticals } \\
\text { - Need for preparation with organic iodine in order to block } \\
\text { the thyroid gland before the procedure } \\
\text { - Procedure } 24-72 \text { hours after tracer administration }\end{array}$ \\
\hline \multicolumn{3}{|c|}{$\begin{array}{l}\text { EUS - endoscopy ultrasound; EUS-FNA - EUS fine-needle aspiration; EUS-RFA - EUS-guided radiofrequency ablation; CEUS - contrast-enhanced } \\
\text { ultrasonography, IDUS - intraductal ultrasonography; IOUS - intraoperative ultrasonography; SRS - somatostatin receptor scintigraphy; SSA - } \\
\text { somatostatin analog; PRRT - peptide-receptor radionuclide therapy; }{ }^{99} \mathrm{mTC} \text {-SPECT - }{ }^{99} \mathrm{mTC} \text {-single-photon emission computed tomography; }{ }^{68} \mathrm{Ga}-\mathrm{DOTA} \text { - } \\
\text { PET - }{ }^{68} \mathrm{Ga} \text {-DOTA-positron emission tomography; }{ }^{18} \mathrm{FDG} \text {-PET - }{ }^{18} \mathrm{FDG} \text {-positron emission tomography; }{ }^{131} \mathrm{I} \text {-MIBG - }{ }^{131} \text { I-MIBG - single-photon emission } \\
\text { computed tomography }\end{array}$} \\
\hline
\end{tabular}

Tab. 2. Proposed diagnostic methods for GEP-NEN $(1,2,18,19)$

\section{Suspected NEN of the stomach, duodenum}

\section{Gastroscopy with histopathological examination (determination of histopathological diagnosis)}

2. EUS (assessment of intramural invasion depth the presence of metastasis in regional lymph nodes) - lesions of 1-2 cm or multiple lesions

3. Abdominal CT scan - after filling the stomach with water to the full (stomach assessment) or 2-stage water drinking (assessment of the duodenum) and i.v. contrast administration / contrast-enhanced MRI - disease staging, distant metastasis detection

4. SRS - disease staging, distant metastasis detection

\section{Suspected NEN of the pancreas}

1. EUS - in every case with clinical diagnosis of a secreting tumour and when indications for biopsy are present

2. Hormonally active NEN of the pancreas - SRS (detection of lesions not revealed using anatomical imaging, search for the primary lesion and determination of the actual stage of the neoplasm; first-line method for the diagnosis of early recurrence, disease monitoring and selecting the right therapy), subsequently EUS and CT/MRI (assessment of anatomical location and the possibility to remove the primary lesion, cancer staging and treatment response monitoring)

3. Hormonally inactive NEN of the pancreas: CT/MRI (as above), subsequently SRS (as above)

4. Rapidly growing NEC and NEN of the pancreas $-{ }^{18} \mathrm{FDG}$ PET/CT Suspected NEN of the small intestine, metastatic NEN of unknown point of origin

1. SRS - method preferred for lesions smaller than $1 \mathrm{~cm}$, search for the primary lesion

2. CT, MRI - search for the primary lesion, disease staging and assessment of treatment response

3. CT or MRI enterography/enteroclysis - determination of location

4. Colonoscopy with distal ileum assessment - search for the primary lesion and exclusion of concomitant cancer (colon cancer)

5. Video capsule endoscopy (VCE) and balloon enteroscopy or spiral enteroscopy - direct assessment of the mucous membrane; poorly available procedure

6. EUS - no utility for small intestinal lesion diagnosis

\section{Suspected NEN of the colon}

1. Colonoscopy - procedure of choice in the diagnosis of colon tumours

2. EUS - in rectal NEN of $\geq 5 \mathrm{~mm}$; ultrasound miniprobes during colonoscopy - in colon tumours diagnosed as polyps/submucosal lesions

3. Abdominal and pelvic CT/MRI with the gastrointestinal tract filled with negative contrast - disease staging and assessment of metastases

4. CT colonography - it is not possible to perform complete colonoscopy in the case of lesions which fully obstruct the intestinal lumen

5. SRS - staging of the neoplastic process, assessment for SSA and PRRT

6. PET-CT following ${ }^{18}$ F-DOPA assessment - in the case of a negative SRS result assessment for antiproliferative treatment using SSA and PRRT

7. ${ }^{18} \mathrm{FDG}-\mathrm{PET} / \mathrm{CT}$ - in patients with NEC, patients with a rapidly growing NET with a negative SRI result and in patients assessed for radioisotope treatment

EUS - endoscopy ultrasound; EUS-FNA - EUS fine-needle aspiration; EUS-RFA - EUS-guided radiofrequency ablation; CEUS - contrast-enhanced ultrasonography; IDUS - intraductal ultrasonography; IOUS - intraoperative ultrasonography; SRS - somatostatin receptor scintigraphy; SSA - somatostatin analog; PRRT - peptide-receptor radionuclide therapy; ${ }^{99} \mathrm{mTc}$-SPECT - ${ }^{99} \mathrm{mTc}$-single-photon emission computed tomography; ${ }^{68} \mathrm{Ga}$-DOTA-PET - ${ }^{68} \mathrm{Ga}-$ -DOTA-positron emission tomography; ${ }^{18}$ FDG-PET - ${ }^{18}$ FDG-positron emission tomography; ${ }^{131}$ I-MIBG - ${ }^{131}$ I-MIBG - single-photon emission computed tomography 
(even 1-2 mm), particularly those hormonally active and a screening method for patients with genetic abnormalities (e.g. with MEN1 syndrome)(4). In a multi-centre retrospective study investigating the size of pancreatic NEN in patients with MEN1 syndrome using EUS more than $90 \%$ of small lesions of less than $7 \mathrm{~mm}$ were visualised ${ }^{(16)}$. In other studies the sensitivity of EUS depended on the location of the focal lesion and was $77-100 \%$ for tumours located in the head and body of the pancreas and 75-80\% for tumours located in the distal part of the pancreas ${ }^{(1,17)}$. Recent studies report that the sensitivity of EUS may be higher than that of computed tomography $(91.7 \%$ vs $63.3 \%$ ), particularly for insulinoma $(84.2 \% \text { vs } 31.6 \%)^{(1,2,4)}$. The sensitivity of EUS in the detection of doudenal lesions is $45-60 \% \%^{(1,2)}$. Comparative studies on conventional examination methods (transabdominal ultrasound, CT and MRI) demonstrated their lower sensitivity compared to EUS ${ }^{(16)}$.

Hormonally active pancreatic neuroendocrine tumours present as single homogeneous, hypoechoic solid tumours (less often normo- and hyperechoic ones) which are characterised by intensive enhancement following contrast administration $^{(16)}$. A cystoid form of the tumour may occur in approximately $10-20 \%$ of patients ${ }^{(18)}$. Calcifications in hormonally active and inactive NEN of the pancreas may be found in approximately $25 \%$ of cases, while being rarely observed in adenocarcinomas. In larger tumours, the pancreatic duct may become dilated and the pancreatic parenchyma may undergo atrophy ${ }^{(1,18)}$. EUS plays an important role in disease staging, assessment of blood vessel invasion and differential diagnosis of pancreatic tumours of unclear nature (distinguishing between NEN and adenocarcinomas, cystoid lesions and tumours with atypical, poor vasculature). It may be stated that EUS combined with Doppler ultrasound, contrast, elastography and EUS-guided biopsy makes it possible to detect all pancreatic $\mathrm{NEN}^{(1,18)}$.

In diagnostic imaging of rectal tumours endoscopic ultrasound is also the most sensitive method for preoperative local staging. A neuroendocrine neoplasm in the form of a polyp presents as a well-defined, iso- or hypoechoic, homogeneous lesion on EUS ${ }^{(19)}$. The sensitivity of this method in the assessment of the tumour and the depth of its invasion in the rectal wall is 76-93\%. For the assessment of metastases in the nearby lymph nodes the sensitivity is $61-88 \% \%^{(1,2,4,19)}$

The assessment of lesions located in the distal part of the ileum is possible on EUS using high-frequency miniprobes inserted through the biopsy channel of a colonoscope ${ }^{(4,19)}$.

An advantage of EUS is also the fact that it is possible to insert a radiofrequency ablation (RFA) probe in the echoendoscope channel, which causes thermal coagulation necrosis of the tumour. It has been demonstrated that this technique is safe and effective in patients with hormonally active NEN of the pancreas who were denied surgery due to comorbidities ${ }^{(13)}$. The disadvantages of EUS include the fact that the result depends on the skills and experience of the examiner and that the field of vision is limited, which may reduce its efficacy in detecting lesions beyond the area of interest ${ }^{(4)}$.

I $t$ is possible to achieve an even better visualisation of pancreatic NEN in the immediate vicinity of the pancreatic duct and abnormalities in its lumen by inserting a miniprobe to the pancreatic or bile duct through the biopsy channel of an endoscope (IDUS) ${ }^{(1)}$. The sensitivity of the procedure is around $94 \%$ and approximates $100 \%$ if the abnormalities in the pancreatic duct are larger than $3 \mathrm{~mm}^{(20)}$.

\section{Contrast-enhanced ultrasound (CEUS)}

Third-generation contrast agents used for ultrasound diagnosis are gas-filled microvesicles coated with phospholipids with a long half-life and perfusion-dependent greyscale image enhancement ${ }^{(1)}$. On CEUS, focal lesions are assessed based on characteristic vasculature patterns indicating benign or malignant abnormality ${ }^{(15)}$. Primary pancreatic NEN, even small ones, have a good network of blood vessels. Following contrast administration, pancreatic NEN show intensive enhancement in the arterial phase $^{(16)}$. The majority of NEN metastases in the liver (78$86 \%$ ) show enhancement in the arterial phase following contrast administration with a quick contrast clearance in the venous phase $\mathrm{e}^{(1,16,17)}$. Wen-Tao et al. demonstrated that metastases with a rich vasculature demonstrated a longer contrast clearance time compared to those with a poorer vasculature. The assessment of both primary and metastatic tumours using CEUS may replace other contrast-enhanced imaging techniques in the majority of cases, particularly if there are contraindications to those procedures ${ }^{(16)}$. CEUS is useful in the detection of small tumours of less than $2 \mathrm{~cm}$, with a comparable sensitivity to EUS $(95 \%)^{(1)}$.

\section{Elastography}

Ultrasound imaging using elastography is an increasingly available technique allowing one to assess tumour hardness. This method may be used to assess focal lesions in the pancreas on EUS and focal lesions of the liver on transabdominal ultrasound. In a study by Iglesias-Garcia et al., the sensitivity of quantitative EUS elastography in differentiating between pancreatic adenocarcinoma and NET was $100 \%$ and its specificity was $88 \%$ for the strain ratio (SR) cut-off value of $26.6^{(21,22)}$. In general, the sensitivity and specificity of differentiating between benign and malignant lesions were $93 \%$ and $86 \%$, respectively, while the SR limit value was 6.0. In a metanalysis on qualitative EUS elastography in the diagnosis of pancreatic tumours a high sensitivity (95\%) and an acceptable low specificity $(67 \%)$ were observed ${ }^{(21,22)}$. Qualitative and quantitative EUS elastography may be a valuable supplementary method used to differentiate between benign and malignant pancreatic lesions ${ }^{(21)}$. According to the recommendations of the European Federation of Societies for Ultrasound in Medicine and Biology (EFSUMB), EUS elastography may be helpful in making therapeutic decisions in cases 
where fine-needle biopsy performed during EUS has no diagnostic value. However, elastography alone cannot replace cytological assessment ${ }^{(21,22)}$. Elastography of focal hepatic lesions allows one to make a preliminary distinction between MEN metastases and primary benign focal hepatic lesions. However, the results may be doubtful in obese patients or in the case of lesions located near the $\operatorname{ribs}^{(21,22)}$.

\section{Intraoperative ultrasound}

Intraoperative ultrasound (IOUS) is used mainly to diagnose focal lesions in the liver and pancreas, particularly in patients with multiple endocrine neoplasia (MEN) syndromes. The procedure also makes it possible to detect multifocal tumours and metastatic lesions in the liver and assess the distance of a pancreatic tumour, particularly a small one, from the pancreatic duct ${ }^{(1)}$. The sensitivity of IOUS in the detection of tumours is $90 \%{ }^{(1)}$.

\section{Conclusion}

Transabdominal ultrasound is usually the first diagnostic imaging procedure for NEN. However, the possibility of precise staging of the disease using transabdominal ultrasound is limited due to a number of factors (technical aspects of the procedure, small tumour dimensions and often unfavourable location inaccessible to transabdominal ultrasound). New ultrasound techniques improve the precision of the diagnosis and constitute an important part of a NEN diagnostic process.

Patients with NEN may show a large discrepancy between the clinical presentation and actual stage of the disease.

\section{References}

1. Kos-Kudła B, Blicharz-Dorniak J, Strzelczyk J, Bałdys-Waligórska A, Bednarczuk T, Bolanowski M et al.: Diagnostic and therapeutic guidelines for gastro-entero-pancreatic neuroendocrine neoplasms (recommended by the Polish Network of Neuroendocrine Tumours). Endokrynol Pol 2017; 68: 79-110.

2. Bodei L, Sundin A, Kidd M, Prasad V, Modlin IM: The status of neuroendocrine tumor imaging: from darkness to light? Neuroendocrinology 2015; 101: 1-17.

3. Maxwell JE, Howe JR: Imaging in neuroendocrine tumors: an update for the clinician. Int J Endocr Oncol 2015; 2: 159-168.

4. Sahani DV, Bonaffini PA, Fernández-Del Castillo C, Blake MA: Gastroenteropancreatic neuroendocrine tumors: role of imaging in diagnosis and management. Radiology 2013; 266: 38-61.

5. Hassan MM, Phan A, Li D, Dagohoy CG, Leary C, Yao JC: Risk factors associated with neuroendocrine tumors: A U.S.-based case-control study. Int J Cancer 2008; 123: 867-873.

6. Lawrence B, Gustafsson BI, Chan A, Svejda B, Kidd M, Modlin IM: The epidemiology of gastroenteropancreatic neuroendocrine tumors. Endocrinol Metab Clin North Am 2011; 40: 1-18.

7. Toumpanakis CG, Caplin ME: Molecular genetics of gastroenteropancreatic neuroendocrine tumors. Am J Gastroenterol 2008; 103: 729-732.

8. Shehata BM, Stockwell CA, Castellano-Sanchez AA, Setzer S, Schmotzer CL, Robinson H: Von Hippel-Lindau (VHL) disease: an
Due to their rare occurrence, atypical symptoms and slow growth, the majority of NEN are diagnosed with a few years' delay, usually at an advanced stage. Only some neuroendocrine tumours present with characteristic signs of hormonal activity, suggesting the diagnosis ${ }^{(1,12,18,19)}$.

Sometimes in asymptomatic patients or patients undergoing ultrasound examination for non-specific abdominal pain a primary lesion is found in the pancreas, less often in the stomach, duodenum or the retroperitoneal space. Metastatic foci in the liver or lymph nodes may also be found. The search for the primary lesion in a patient with metastatic NEN of an unknown point of origin may be particularly difficult since primary lesions are often small. Depending on the type of visualised changes the examiner should propose a further diagnostic algorithm.

In a reverse situation when NEN is diagnosed based on clinical symptoms and/or biochemical testing, the key steps are to search for the primary lesions and make a baseline assessment of the stage of the neoplastic process. Depending on the symptoms and the type of the suspected NEN one should identify the possible sites of the tumour focusing on the pancreas, intestines, appendix, mesentery and retroperitoneal space (Tab. 2).

\section{Conflict of interest}

The authors do not report any financial or personal affiliations to persons or organisations that could adversely affect the content of or claim to have rights to this publication.

update on the clinico-pathologic and genetic aspects. Adv Anat Pathol 2008; 15: 165-171.

9. Lodish MB, Stratakis CA: Endocrine tumours in neurofibromatosis type 1, tuberous sclerosis and related syndromes. Best Pract Res Clin Endocrinol Metab 2010; 24: 439-449.

10. Tsujimura K, Takushi Y, Teruya T, Iha K, Ota M, Nakachi A et al.: Neuroendocrine tumor of the small intestine diagnosed with trans-abdominal ultrasonography: A case report. Int J Surgery Case Reports 2017; 31: 75-78.

11. Miller HC, Frampton AE, Malczewska A, Ottaviani S, Stronach EA, Flora R et al.: MicroRNAs associated with small bowel neuroendocrine tumours and their metastases. Endocr Relat Cancer 2016; 23: 711-726.

12. Bednarczuk T, Bolanowski M, Zemczak A, Bałdys-Waligórska A, Blicharz-Dorniak J, Boratyn-Nowicka A et al.: Neuroendocrine neoplasms of the small intestine and the appendix - management guidelines (recommended by the Polish Network of Neuroendocrine Tumours). Endokrynol Pol 2017; 68: 223-236.

13. Tan $\mathrm{EH}$, Tan $\mathrm{CH}$ : Imaging of gastroenteropancreatic neuroendocrine tumors. World J Clin Oncol 2011; 2: 28-43.

14. Kong WT, Ji ZB, Wang WP, Cai H, Huang BJ, Ding H: Evaluation of liver metastases using contrast-enhanced ultrasound: enhancement patterns and influencing factors. Gut Liver 2016; 10: 283-287.

15. Braden B, Jenssen Ch, D'Onofrio M, Hocke M, Will U, Möller K et al.: $\mathrm{B}$-mode and contrast-enhancement characteristics of small noninci- 
dental neuroendocrine pancreatic tumors. Endosc Ultrasound 2017; 6: 49-54.

16. Dietrich CF, Ignee A, Braden B, Barreiros AP, Ott M, Hocke M: Improved differentiation of pancreatic tumors using contrast-enhanced endoscopic ultrasound. Clin Gastroenterol Hepatol 2008; 6: 590-597.

17. Chiti A, Fanti S, Savelli G, Romeo A, Bellanova B, Rodari M et al.: Comparison of somatostatin receptor imaging, computed tomography and ultrasound in the clinical management of neuroendocrine gastroentero-pancreatic tumours. Eur J Nucl Med 1998; 25: 1396-1403.

18. Kos-Kudła B, Rosiek V, Borowska M, Bałdys-Waligórska A, Bednarczuk T, Blicharz-Dorniak J et al.: Pancreatic neuroendocrine neoplasms - management guidelines (recommended by the Polish Network of Neuroendocrine Tumours). Endokrynol Pol 2017; 68: 169-197.
19. Starzyńska T, Londzin-Olesik M, Bałdys-Waligórska A, Bednarczuk T, Blicharz-Dorniak J, Bolanowski M et al.: Colorectal neuroendocrine neoplasms - management guidelines (recommended by the Polish Network of Neuroendocrine Tumours). Endokrynol Pol 2017; 68: 250-260.

20. Sun Bo, Hu B: The role of intraductal ultrasonography in pancreatobiliary diseases. Endosc Ultrasound 2016; 5: 291-299.

21. Nemakayala D, Patel P, Rahimi E, Fallon MB, Thosani N: Use of quantitative endoscopic ultrasound elastography for diagnosis of pancreatic neuroendocrine tumors. Endosc Ultrasound 2016; 5: 342-345.

22. Iglesias-Garcia J, Larino-Noia J, Abdulkader I, Forteza J, DominguezMunoz JE: Quantitative endoscopic ultrasound elastography: An accurate method for the differentiation of solid pancreatic masses. Gastroenterology 2010; 139: 1172-1180. 